\title{
Whole-genome approach implicates CD44 in cellular resistance to carboplatin
}

\author{
Sunita J. Shukla,' Shiwei Duan, ${ }^{2}$ Xiaolin Wu, ${ }^{2}$ Judith A. Badner, ${ }^{3}$ Kristen Kasza ${ }^{4}$ and M. Eileen Dolan ${ }^{2,5,6 *}$ \\ 'Department of Human Genetics, University of Chicago, Chicago, IL 60637, USA \\ ${ }^{2}$ Department of Medicine, University of Chicago, Chicago IL 60637, USA \\ ${ }^{3}$ Department of Psychiatry, University of Chicago, Chicago IL 60637, USA \\ ${ }^{4}$ Department of Health Studies, University of Chicago, Chicago IL 60637, USA \\ ${ }^{5}$ Committee on Cancer Biology, University of Chicago, Chicago IL 60637, USA \\ ${ }^{6}$ Committee on Clinical Pharmacology and Pharmacogenomics, University of Chicago, Chicago IL 60637, USA \\ *Correspondence to: Tel: +1 773 702-4441; Fax: +1 773702 0963; E-mail: edolan@medicine.bsd.uchicago.edu
}

Date received (in revised form): 22nd September, 2008

\begin{abstract}
Carboplatin is a chemotherapeutic agent used in the management of many cancers, yet treatment is limited by resistance and toxicities. To achieve a better understanding of the genetic contribution to carboplatin resistance or toxicities, lymphoblastoid cell lines from 34 large Centre d'Etude du Polymorphisme Humain pedigrees were utilised to evaluate interindividual variation in carboplatin cytotoxicity. Significant heritability, ranging from 0.17-0.36 $\left(\mathrm{p}=\mathrm{I} \times 10^{-7}\right.$ to $\left.9 \times 10^{-4}\right)$, was found for cell growth inhibition following 72-hour treatment at each carboplatin concentration $(10,20,40$ and $80 \mu \mathrm{M})$ and $\mathrm{IC}_{50}$ (concentration for 50 per cent cell growth inhibition). Linkage analysis revealed II regions with logarithm of odds (LOD) scores greater than I.5. The highest LOD score on chromosome II $\left(\mathrm{LOD}=3.36, \mathrm{p}=4.2 \times 10^{-5}\right)$ encompasses 65 genes within the I LOD confidence interval for the carboplatin $\mathrm{IC}_{50}$. We further analysed the $\mathrm{IC}_{50}$ phenotype with a linkage-directed association analysis using 7I unrelated HapMap and Perlegen cell lines and identified I8 single nucleotide polymorphisms within eight genes that were significantly associated with the carboplatin $I C_{50}\left(p<3.6 \times 10^{-5}\right.$; false discovery rate $<5$ per cent). Next, we performed linear regression on the baseline expression and carboplatin $I C_{50}$ values of the eight associated genes, which identified the most significant correlation between CD44 expression and $I C_{50}\left(r^{2}=0.20 ; p=6 \times 10^{-4}\right)$. The quantitative real-time polymerase chain reaction further confirmed a statistically significant difference in CD44 expression levels between carboplatin-resistant and -sensitive cell lines $\left(p=5.9 \times 10^{-3}\right)$. Knockdown of CD44 expression through small interfering RNA resulted in increased cellular sensitivity to carboplatin $(p<0.01)$. Our whole-genome approach using molecular experiments identified CD44 as being important in conferring cellular resistance to carboplatin.
\end{abstract}

Keywords: CD44, carboplatin, CEPH, HapMap, expression, linkage

\section{Introduction}

The antitumour effects of platinating agents have contributed significantly to the clinical management of a variety of cancers, including ovarian, head and neck, and non-small cell lung carcinomas. ${ }^{1,2}$ These agents exert their antitumour activity by binding preferentially to the N-7 positions of adenine and guanine of DNA, resulting in the formation of intra- and inter-strand cross-links. Cisplatin and carboplatin (Figure 1) have similar mechanisms of action; differences in potency between the two drugs relate to different aquation rates. Although cisplatin has had a major clinical impact, carboplatin, with its more stable leaving group, was developed as a less toxic analogue that retained its antitumor activity. ${ }^{2}$ In 1989, Food and Drug Administration approval was granted for a 
carboplatin-based regimen as the standard of care for ovarian cancer. ${ }^{2}$ As seen with cisplatin, intrinsic and/or acquired resistance, as well as toxicities, associated with carboplatin are major limitations of this drug. ${ }^{1,3,4}$ Carboplatin resistance may be multifactorial, consisting of increased efflux from the cell, drug inactivation, increased DNA repair and evasion of apoptosis. ${ }^{5,6}$ Candidate genes that may be involved in resistance to carboplatin are illustrated in the platinum-based pathway on the PharmGKB website (http://www.pharmgkb.org).

The major dose-limiting toxicity associated with carboplatin is myelosuppression. ${ }^{7}$ Specifically, carboplatin can cause thrombocytopenia in 20-40 per cent of patients and severe neutropenia in approximately 20 per cent of treated patients. ${ }^{8}$ A few association studies evaluating single nucleotide polymorphisms (SNPs) within candidate genes have been performed with regard to platinum-based clinical response and outcome. Suk et al. ${ }^{9}$ found that the germline $\mathrm{C} / \mathrm{A}$ or $\mathrm{A} / \mathrm{A}$ genotype at the C8092A polymorphism within ERCC1 conferred a 2.33-fold relative increase in the risk of developing severe gastrointestinal toxicity. Several studies have shown that a subset of lung cancer patients whose tumours do not express ERCC1 are likely to receive a survival benefit from adjuvant therapy with a platinum agent, while patients who do express ERCC1 derive no such benefit. ${ }^{10,11}$ In metastatic breast cancer patients, a study identified an association between polymorphisms in XRCC1 and XRCC3 and survival after receiving treatment regimens that included carboplatin. ${ }^{12}$ These findings regarding DNA repair genes emerged as a result of a candidate gene approach, an approach that is limited to genes known to be involved in the mechanism of action of the drug. Drug toxicity and response are probably multigenic traits,

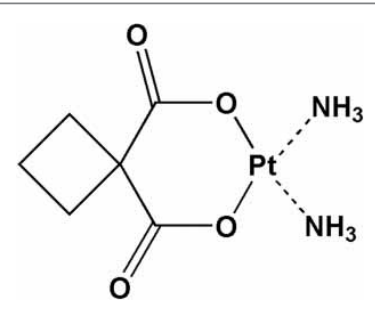

Figure I. Chemical structure of carboplatin. however, and not all genes that may be important are included in such studies.

Our laboratory is focused on building cell-based genetic models to identify genes and variants conferring sensitivity to chemotherapeutic agents. ${ }^{13-17}$ Using lymphoblastoid cell lines (LCLs) from apparently healthy individuals within large Centre d'Etude du Polymorphisme Humain (CEPH) families, we found that chemotherapeutic-induced cytotoxicity is significantly heritable for cisplatin and daunorubicin. ${ }^{13,14,17}$ In the present study, we utilised large $\mathrm{CEPH}$ pedigrees for genome-wide linkage and unrelated Hapmap and Perlegen LCLs for linkagedirected association analysis to identify genes and variants involved in carboplatin-induced cytotoxicity. The identified genes were further interrogated to determine whether baseline gene expression correlated with carboplatin $\mathrm{IC}_{50}$. We successfully performed expression knockdown studies of one such target gene, CD44, whose expression explained a significant proportion of the variation in carboplatin $\mathrm{IC}_{50}$. This finding validated the role of CD44 in carboplatin resistance in these cell lines.

\section{Materials and methods}

\section{Cell lines}

Four hundred and forty-seven Epstein-Barr virus (EBV)-transformed LCLs derived from 34 Caucasian Utah CEPH families of northern and western European descent (1331, 1332, 1333, 1334, 1340, 1341, 1344, 1345, 1346, 1347, 1349, 1350, 1356, 1358, 1362, 1375, 1377, 1408, 1413, 1416, 1418, 1420, 1421, 1423, 1424, 1444, 1447, 1454, 1459, 1463, 13291, 13292, 13293, 13294) were used for heritability and linkage analyses. Fifty-seven out of 60 unrelated cell lines (from the same families) from the HapMap Project (GM06985, GM06993, GM06994, GM07000, GM07022， GM07034， GM07055， GM07056, GM07345， GM07357， GM11829， GM11830, GM11831， GM11832， GM11839， GM11840, GM11881, GM11882, GM11992, GM11993, GM11994, GM11995, GM12003， GM12004, GM12005， GM12006， GM12043， GM12044, GM12056, GM12057, GM12144, GM12145, 
GM12146, GM12154, GM12155， GM12156, GM12234, GM12239, GM12248, GM12249, GM12264， GM12750， GM12751， GM12760, GM12761， GM12762， GM12763， GM12812GM12815, GM12872, GM12873, GM12874, GM12875, GM12891, GM12892) and 11 unrelated cell lines from the Perlegen Project (GM06990, GM07349, GM10842-10845, GM10848, GM10850, GM10852, GM10853, GM10858) were phenotyped for sensitivity to carboplatin. Cells were purchased from Coriell Institute for Medical Research (http://ccr.coriell.org/Sections/Collections/ NIGMS?SsID=8). Two HapMap samples (GM12716 and GM12717) were not phenotyped owing to the inability to obtain $>85$ per cent viability. Additionally, another sample (GM12236) was not phenotyped because it was not available from Coriell at the time of phenotyping, owing to the cell line not meeting all quality control standards at Coriell (eg slow growth). Cell lines were cultured in RPMI 1640 medium containing 15 per cent heat-inactivated foetal bovine serum (Hyclone, Logan, UT, USA) and $20 \mathrm{mM}$ L-glutamine. Cell lines were diluted three times per week at a concentration of $300,000-$ 350,000 cells $/ \mathrm{ml}$ and were maintained in a $37^{\circ} \mathrm{C}, 5$ per cent $\mathrm{CO}_{2}$ humidified incubator. Medium and components were purchased from Cellgro (Herndon, VA, USA).

\section{Drugs}

Cis diamine (1,1-cyclobutanedicarboxylato) platinum (carboplatin) was purchased from Sigma Chemical Co. (St Louis, MO, USA). Figure 1 illustrates the structure of carboplatin. Carboplatin was prepared as a $20 \mathrm{mM}$ stock in water, filter sterilised and diluted in medium immediately prior to addition to cells.

\section{Cell growth inhibition}

Cytotoxicity was assessed in the absence (control) and presence of increasing concentrations of carboplatin, utilising a high-throughput alamarBlue ${ }^{\mathrm{TM}}$ (Biosource International, Camarillo, CA, USA) assay, as previously described. ${ }^{14}$ Final concentrations of carboplatin used for the heritability and linkage analyses were 10, 20, 40 and $80 \mu \mathrm{M}$, with an exposure time of 72 hours. Cytotoxicity measurements were performed in triplicate for each drug concentration per experiment, with two to three experiments per cell line. Final cytotoxicity values were averaged from at least six replicates taken from two separate experiments. $\mathrm{IC}_{50}$, the concentration required to inhibit 50 per cent of cell growth, was calculated for each cell line by curve fitting each concentration using Microsoft Excel ${ }^{\mathrm{TM}}$ software. For cytotoxicity studies following small interfering RNA (siRNA) knockdown, cells were plated one hour after transfection and treated with 20, 40, 80, 160, 320 and $640 \mu \mathrm{M}$ carboplatin for 48 hours to expose cells to drug at the time of greatest CD44 mRNA and protein knockdown. Three independent knockdown experiments were performed in triplicate for each of two cell lines with high CD44 expression (GM06985 and GM11881).

\section{Heritability analysis}

Heritability analysis was performed using Sequential Oligogenic Linkage Analysis Routines (SOLAR; http://www.sfbr.org/solar/) computer software to estimate narrow sense heritability at each carboplatin concentration. ${ }^{18}$ SOLAR uses likelihood ratio tests to evaluate heritability by comparing a purely polygenic model with a sporadic model in the case of testing heritability. This analysis is used to determine whether there is a significant proportion of cytotoxicity at each dose that can be explained by genetic factors. All phenotype data were transformed using the inverse normalisation of the percentile rank function in Microsoft Excel ${ }^{\mathrm{TM}}$ software. Covariates such as age, sex and the age-sex interaction were tested as previously described. ${ }^{14}$

\section{Linkage analysis}

MERLIN $^{19}$ was used to perform non-parametric quantitative trait locus (QTL) linkage analysis, which is robust to non-normally distributed data. The genotypic data were downloaded from the CEPH database (http://www.cephb.fr/cephdb/) and the Marshfield map database (http://research. marshfieldclinic.org/genetics/GeneticResearch/comp Maps.asp). The CEPH database (version 10) contains genotypic information on over 32,000 genetic markers and 9,000 microsatellite markers typed in 
the CEPH LCLs. Overall, this database manages over 6 million genotypes, which can be utilised for genetic studies. The Marshfield map database was used to determine microsatellite locations within particular chromosomal regions of interest. The genetic markers were error checked for Mendelian incompatibility, mis-specified relationship and unlikely recombinations, using a platform for linkage analysis as previously described. ${ }^{13,14}$ The SNP data were downloaded from the SNP Consortium (www.snp.cshl. org). From the combined pool of SNP and microsatellite markers genotyped in the above databases, approximately 7,209 non-redundant markers were selected based on the availability of genotypes in at least 50 per cent of family members. Physical positions of selected microsatellite and SNP markers were found using Build 36 of the UCSC Genome Browser (http://www.genome.ucsc.edu.). Genetic maps were constructed based on microsatellite and SNP positions in the Marshfield map. These highly heterozygous markers, yielding a dense genetic map, were utilised for the analysis. Age and sex were also included as covariates in the analysis.

\section{Association analysis}

SNP genotypes were downloaded from the International HapMap Project (http://hapmap.org) (release 20) and Perlegen (http://perlegen.com) genotype databases for the 68 unrelated CEPH/UTAH (57 HapMap plus 11 Perlegen) samples. To exclude possible genotyping errors in overlapping samples from the HapMap and Perlegen datasets, 23,189 Perlegen SNPs with Mendelian transmission errors and 13,803 SNPs with inconsistent genotypes were removed. A total of 277,000 SNPs, located 5 kilobases upstream/downstream of genes, in addition to within genes, were used for the analysis.

The single-locus analysis was performed using one-way analysis of variance (ANOVA), with Bartlett's test for equal variances cutoff as $p=0.01$, using R-2.3.1. Since multivariate normality of the phenotypic data is a critical assumption of ANOVA, the $\mathrm{IC}_{50}$ phenotype was transformed into normal distributions using $\log _{2}$ transformations. ANOVA was then performed on unrelated samples to assess the non-linear relationship among the genotypes.
False discovery rate (FDR) was used to control for multiple testing within the phenotype using the $Q$ Value package of R-2.3.1 (www.r-project.com). An association $p$ value with an FDR of less than 5 per cent was defined as significant.

\section{Gene expression}

Baseline gene expression values for HapMap CEPH samples were determined as previously described $^{20}$ using the Affymetrix GeneChip Human Exon 1.0 ST array.

\section{Quantitative real-time polymerase chain reaction (qRT-PCR)}

Up to $5 \times 10^{6}$ total number of cells from ten LCLs with the highest carboplatin $\mathrm{IC}_{50}$ values $\left(\mathrm{IC}_{50}\right.$ range: 91.3-34.7) and ten LCLs with the lowest carboplatin $\mathrm{IC}_{50}$ values ( $\mathrm{IC}_{50}$ range: $10.4-13.3$ ) (out of the 68 unrelated HapMap and Perlegen samples) were evaluated for CD44 baseline expression. The LCLs with the lowest $\mathrm{IC}_{50}$ values were: GM10845, GM10853, GM12006, GM12043, GM12145, GM12146, GM12812, GM12813, GM12815 and GM12872. The LCLs with the highest $\mathrm{IC}_{50}$ values were: GM06985, GM11830, GM11881, GM12044, GM12056, GM12751, GM12761 and GM12891. LCLs in exponential growth were pelleted, washed three times with ice cold phosphate-buffered saline, flash frozen in liquid nitrogen, and stored in a freezer at $-80^{\circ} \mathrm{C}$ until required. Total RNA was isolated from the cells using the Qiagen RNeasy Mini kit (Valencia, CA, USA), according to the manufacturer's protocol. Gene expression was quantified with the Roche LightCycler SYBR Green I kit (Indianapolis, IN, USA) and samples were run (one-step PCR) on the 7900 HT Fast Real Time PCR System from Applied Biosciences (Foster City, CA, USA). PCR primers were designed and tested for gene homology using the UCSC Genome Browser (www.genome.ucsc.edu) and BLAST (www.ncbi.nlm.nih.gov/BLAST) programs. Primers were designed to be approximately $20-25$ base pairs in length, have 50-60 per cent GC content and melting temperatures of $55-65^{\circ} \mathrm{C}$. CD44 primers were designed to capture all isoforms with the forward primer 5'-GCATTGCAGTCAACAGTCG 
AAGAAG- $3^{\prime}$ and the reverse primer $5^{\prime}-\mathrm{GG}$ CCTCTCCGTTGAGTCCACTT-3'. Lamin A/C primer sequences included forward primer $5^{\prime}$-ATG ATCGCTTGGCGGTCTAC- $3^{\prime}$ and reverse primer 5'-GCCCTGCGTTCTCCGTTT-3'. Beta-actin was used as an endogenous control with the forward primer 5'-ATTGCCGACAGGATGCAGA-3' and reverse primer $5^{\prime}$-GCTCAGGAGGAGCAATGAG CTT-3'. Standard curves were prepared for all genes, with fivefold dilutions ranging from $2,000 \mathrm{ng} / \mu \mathrm{l}$ to $0.64 \mathrm{ng} / \mu \mathrm{l}$. Standard curves were only used if they had $r^{2}$ values $>0.985$. CD44 and beta-actin were run under identical conditions, with parameters as follows: Step 1: $55^{\circ} \times 1,800$ seconds; Step 2: $95^{\circ} \times$ 600 seconds; Step 3: $95^{\circ} \times$ ten seconds, $58^{\circ} \times 15$ seconds, $72^{\circ} \times 20$ seconds (repeated 45 times). Lamin $A / C$ primers were run under the following conditions: Step 1: $55^{\circ} \times 1,800$ seconds; Step 2: $95^{\circ} \times 600$ seconds; Step 3: $95^{\circ} \times 15$ seconds, $60^{\circ} \times 60$ seconds, $72^{\circ} \times$ six seconds (repeated 45 times). Melt curve analysis was performed for each run. Three separate experiments were run; within each experiment, samples were run in duplicate. Student's $t$-test was used for comparison of normalised CD44 expression levels between cells with high and low carboplatin $\mathrm{IC}_{50}$ values.

\section{siRNA knockdown of CD44}

LCLs (GM06985 and GM11881) were grown to 7$9 \times 10^{5}$ cells $/ \mathrm{ml}$ and transfected 24 hours after diluting with an Amaxa Nucleofector I device, according to the manufacturer's recommendations (Amaxa Biosystems Inc., Gaithersburg, MD, USA). Electrical parameter X-01 and Nucleofector kit $\mathrm{V}$ were used for the single cuvette transfection process. Nucleofected cells were immediately transferred into a flask containing $3 \mathrm{ml}$ medium and then placed in a humidified incubator at $37^{\circ} \mathrm{C}, 5$ per cent $\mathrm{CO}_{2}$. Transfection efficiency, typically between 70 and 80 per cent, was determined by flow cytometry using fluorescently labelled siGLO siRNA directed against lamin $A / C$ from Dharmacon (Lafayette, CO, USA). Transfection efficiency was also assessed by mRNA knockdown of lamin A/C 24 hours post-transfection using qRT-PCR conditions as described above. CD44 On-Targetplus pool siRNA and control
On-Targetplus siCONTROL non-targeting pool siRNA (Dharmacon) were used. All siRNAs were used at equal concentrations $(600 \mathrm{pmol})$. Typical mRNA knockdown of CD44, as compared with non-targeting control, was assessed after 24 hours.

\section{Western blotting}

CD44 protein levels were analysed in total cell extracts prepared by lysing the cells in RIPA buffer in the presence of protease inhibitors (Santa Cruz Biotechnology, Santa Cruz, CA, USA). Protein concentrations were analysed at $595 \mathrm{~nm}$ using Bio-Rad's (Hercules, CA, USA) Bradford protein assay. Since CD44 is highly expressed in LCLs, $1 \mu \mathrm{g}$ total protein was separated on a 4-20 per cent tris-glycine gel (Bio-Rad) under non-reducing conditions and transferred to a polyvinylidene difluoride (PVDF) membrane. The membrane was blocked for at least one hour in 3 per cent milk. Primary CD44 (recognising the extracellular $\mathrm{N}$-terminal domain) and $\beta$-actin antibodies (Abcam, Cambridge, MA, USA) were added in 1:5,000 dilutions overnight at $4^{\circ} \mathrm{C}$. Proteins were visualised using horseradish peroxidase-conjugated secondary antibodies (1:10,000 dilutions) from Millipore (St Charles, MO, USA) and ECL plus Western Blotting Detection Reagent (General Electric Healthcare, Piscataway, NJ, USA). CD44 protein levels were measured 48-96 hours after siRNA transfection.

\section{Statistical analysis}

$\mathrm{IC}_{50}$ values, from at least three separate knockdown experiments, were compared using Student's $t$-test. To improve the statistical power, the cellular survival results across the two cell lines (GM06985 and GM11881) were combined by performing a mixed model ANOVA, with cell line as a random effect and dose and experimental condition (CD44 siRNA versus non-targeting group) as fixed effects. The interaction between dose and experimental condition was also tested.

\section{Results}

\section{Carboplatin-induced cytotoxicity}

Lymphoblastoid cell lines derived from 34 large CEPH pedigrees (447 total cell lines) were evaluated for cell growth inhibition following exposure 
to increasing concentrations of carboplatin for 72 hours. The mean $( \pm S D)$ percentage cell survival pertaining to the $10,20,40$ and $80 \mu \mathrm{M}$ carboplatin doses were $65.7 \pm 8.7,52.7 \pm 9.87,39.8 \pm 9.59$ and $27.1 \pm 9.17$ per cent, respectively. $\mathrm{IC}_{50}$ was determined for 94 per cent $(422 / 447)$ of cell lines because of the requirement to have survival values above and below 50 per cent. The mean $\mathrm{IC}_{50}$ $( \pm \mathrm{SD})$ concentration was $25.8 \pm 13 \mu \mathrm{M}$, with a range of $8.28-91.3 \mu \mathrm{M}$. Intra- and interfamily variations for all of the carboplatin concentrations are shown in the box plots in Figure 2.

\section{Heritability analysis}

Heritability analysis, which compares the covariance of each trait with the overall variance of that trait, revealed a significant genetic contribution for the cytotoxic effects of each carboplatin drug concentration (Figure 2). Approximately 17-36 per cent $\left(p=1 \times 10^{-7}\right.$ to $\left.9 \times 10^{-4}\right)$ of the variation in carboplatin cytotoxicity was due to genetic factors, with no sex-specific effects for any of the phenotypes tested. The heritability for the $\mathrm{IC}_{50}$ phenotype was $0.21\left(p=2.7 \times 10^{-5}\right)$.

\section{Linkage analysis}

Non-parametric QTL linkage analysis was performed on all four carboplatin concentrations, as well as the $\mathrm{IC}_{50}$ phenotype. There were 11 different genomic regions (containing 1,212 non-redundant genes) with LOD scores $>1.5$ for the different carboplatin concentrations tested (Table 1, Figure 3). The highest LOD score of 3.36 was found on chromosome 11q14.3-11q23.3 for the $\mathrm{IC}_{50}$ phenotype, with 151 genes within the 1 LOD confidence interval region. This region also contained a suggestive linkage peak corresponding to the $10 \mu \mathrm{M}$ concentration, with a LOD score of 1.57. Other overlapping linkage regions included chromosome 8 for the 20, 40 and $80 \mu \mathrm{M}$ concentrations and $\mathrm{IC}_{50}$, with associated LOD scores of 1.65, 1.61, 2.09 and 1.87, and chromosome 9 for the 40 and $80 \mu \mathrm{M}$ concentrations, with associated LOD scores of 1.58 and 1.59 , respectively.

\section{Association analysis}

Since the carboplatin $\mathrm{IC}_{50}$ phenotype was associated with the highest linkage peak (Figure 3), we chose this phenotype for the next step in our analysis. There were over 440 genes underneath the suggestive peaks, with LOD $>1.5$ for the carboplatin $\mathrm{IC}_{50}$. To prioritise SNPs within this region, we performed an ANOVA-based linkage-directed association analysis on a subset of samples within the pedigrees that had publicly available dense genotypic data - specifically, unrelated HapMap and Perlegen samples. This analysis resulted in 18 SNPs, corresponding to eight genes (located on chromosomes 8 and 11) that were significantly associated with the $\mathrm{IC}_{50}$ phenotype (Table 1, Supplementary Table 1). The associated genes were: BAALC, C8orf76, CD44, CNTN5, DKFZP586H2123, IGSF4, LRRC4C and PAK1. The highest FDR value for the SNPs contained within the associated genes was 2.7 per cent (Supplementary Table 1). Genes belonging to the biological process of cell adhesion (CD44, CNTN5, IGSF4), as determined by Entrez Gene (www. http://www.ncbi.nlm.nih.gov/sites/entrez?db=gene), were represented as significantly associated with the carboplatin $\mathrm{IC}_{50}$ and were located underneath the linkage peak on chromosome 11 (LOD $=2.54$ ).

\section{Baseline gene expression correlation with carboplatin $\mathrm{IC}_{50}$}

To examine the contribution of baseline gene expression of the eight associated genes to the carboplatin $\mathrm{IC}_{50}$, we utilised our previously published Affymetrix exon array expression data on the HapMap cell lines. ${ }^{16} B A A L C, L R R C 4 C$, DKFZP586H2123 and CNTN5 were not expressed at baseline (data not shown). Of the remaining genes, linear regression was performed using the $\log _{2}$-transformed expression and $\mathrm{IC}_{50}$ values of the HapMap unrelated cell lines. The most significant correlation was observed with CD44, in which 20 per cent $\left(p=6 \times 10^{-4}\right)$ of the variation in carboplatin $\mathrm{IC}_{50}$ was explained by CD44 baseline expression, with higher CD44 expression corresponding to carboplatin resistance (Figure $4 \mathrm{a}$ ). Baseline PAK1 expression explained 9 per cent of the variation in carboplatin $\mathrm{IC}_{50}\left(\mathrm{p}=2 \times 10^{-2}\right)$ but IGSF4 and C8orf76 baseline expression did not explain a significant amount of variation in carboplatin $\mathrm{IC}_{50}(p>0.05)$. Since CD44 expression 


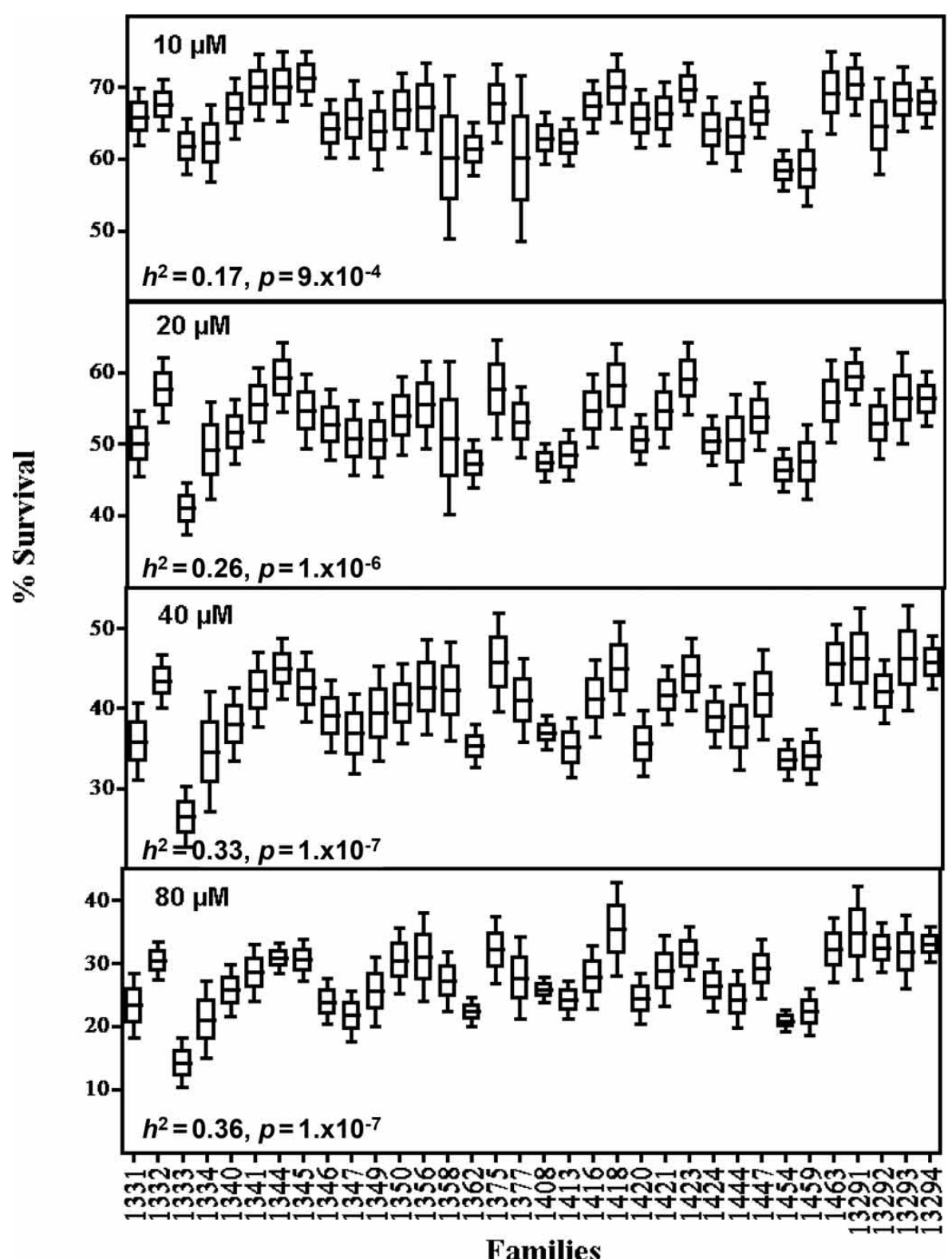

Figure 2. Box plots and heritability $\left(h^{2}\right)$ values for 34 families are shown for 10, 20, 40 and $80 \mu M$ carboplatin. Centre d'Etude du Polymorphisme Humain (CEPH) family identification pertaining to the box plot is located on the x-axis. The middle line within the box represents the mean percentage survival of each family, while the box edges and whiskers represent the standard error of the mean and twice the standard error of the mean, respectively.

explained the most variation in carboplatin $\mathrm{IC}_{50}$, the relationship between CD44 baseline expression and carboplatin $\mathrm{IC}_{50}$ was further investigated.

\section{qRT-PCR of CD44 on resistant and sensitive LCLs}

To validate the relationship of CD44 expression to cytotoxicity identified using exon array, qRT-PCR was performed on eight LCLs with the highest $\mathrm{IC}_{50}$ values (range: 91.3-34.7) and ten LCLs with the lowest $\mathrm{IC}_{50}$ values (range: 10.4-13.3). The mean normalised CD44 expression value of the resistant LCLs was 2.3-fold higher than that of sensitive cells (Student's t-test: $p=5.9 \times 10^{-3}$ ), consistent with exon array data, indicating that CD44 expression confers resistance to carboplatin 


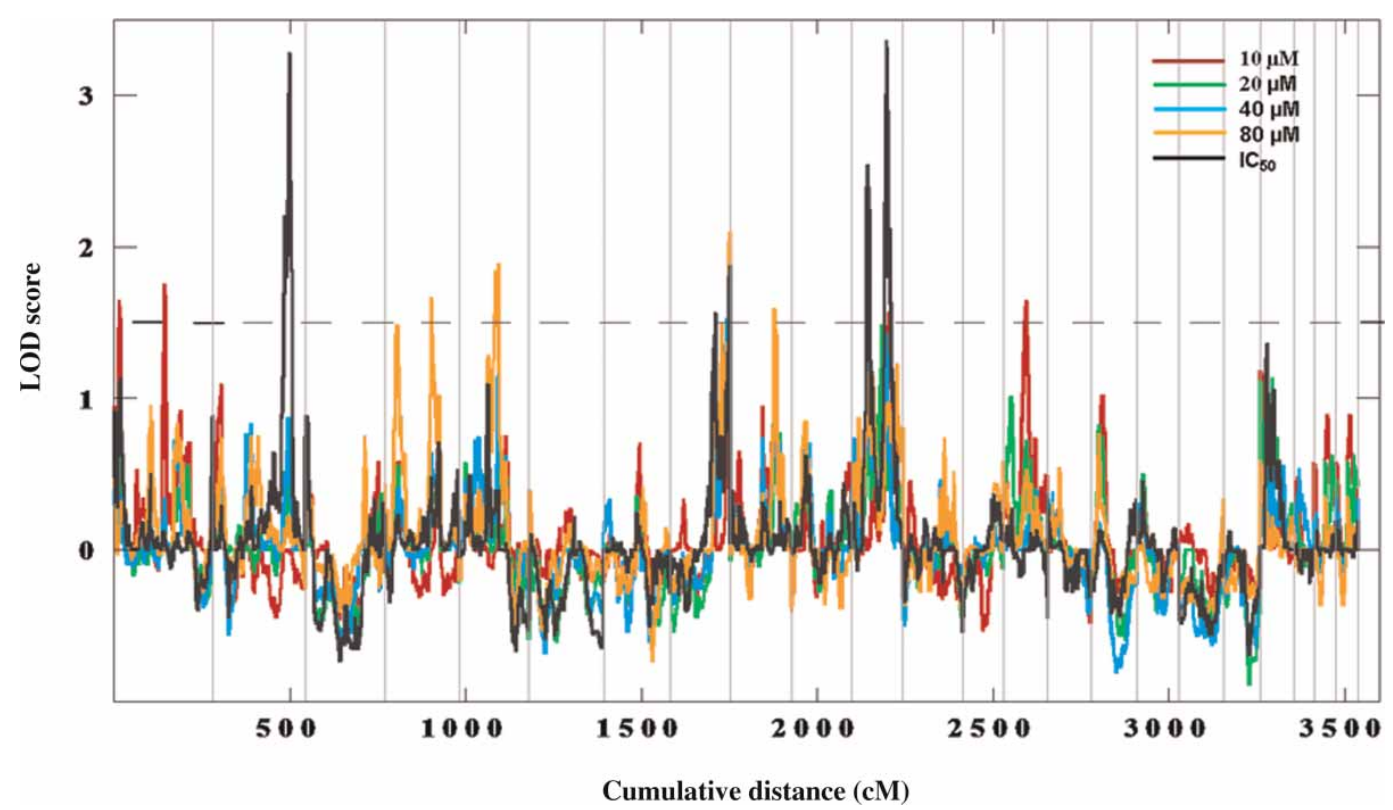

Figure 3. Linkage analyses for carboplatin-induced cytotoxicity. Non-parametric quantitative trait locus (QTL) linkage analysis was performed using 34 large pedigrees for the 10,20, 40 and $80 \mu \mathrm{M}$ concentrations and $I_{50}$ phenotype. Each vertical line indicates chromosome boundaries and the horizontal lines indicate logarithm of odds (LOD) scores. The dotted line indicates the LOD $>$ I.5 regions, which were used for association analyses.

Table I. Genes overlapping linkage and association analyses for carboplatin

\begin{tabular}{|c|c|c|c|c|c|c|}
\hline $\begin{array}{l}\text { I LOD } \\
\text { confidence } \\
\text { interval }\end{array}$ & $\begin{array}{l}\text { I LOD } \\
\text { confidence } \\
\text { interval } \\
\text { (cM) }\end{array}$ & $\begin{array}{l}\text { Max } \\
\text { LOD } \\
\text { (cM) }\end{array}$ & $\begin{array}{l}\text { Phenotype } \\
(\mu M)\end{array}$ & $\begin{array}{l}\text { No. of } \\
\text { genes } \\
\text { in I } \\
\text { LOD } \\
\text { interval }\end{array}$ & $\begin{array}{l}\text { Associated } \\
\text { gene; SNP } \\
\left(^{(p \text { value })^{a}}\right.\end{array}$ & Function \\
\hline Ip36.32-36.22 & $6-23$ & $1.64(15)$ & 10 & 69 & & \\
\hline Ip2I.I-p|3.I & $137-150$ & 1.75 (144) & 10 & 130 & & \\
\hline $2 q 33.3-q 36.3$ & $203-228$ & $3.28(220)$ & $I C_{50}$ & 127 & & \\
\hline 4q27-q32.I & $127-157$ & 1.66 (I33) & 80 & 118 & & \\
\hline $5 q 14.3-q 22.1$ & $95-116$ & $1.88(112)$ & 80 & 63 & & \\
\hline $8 q 22.2-q 24.21$ & $114-136$ & $1.56(128)$ & $I C_{50}$ & 92 & $\begin{array}{l}\text { BAALC; } \\
\text { rs2201369 } \\
\left(9.12 \times 10^{-11}\right)\end{array}$ & $\begin{array}{l}\text { Neuroectoderm/ } \\
\text { haematopoietic } \\
\text { development }\end{array}$ \\
\hline \multirow[t]{4}{*}{$8 q 24.13-q 24.22$} & $158-170$ & 2.09 (169) & 80 & 26 & & \\
\hline & $158-170$ & 1.87 (169) & $I C_{50}$ & 26 & $\begin{array}{l}\text { C8orf76; } \\
\text { rs I7378130 } \\
\left(1.48 \times 10^{-5}\right)\end{array}$ & \\
\hline & $154-170$ & 1.65 (165) & 20 & 30 & & \\
\hline & $155-170$ & I.6I (168) & 40 & 27 & & \\
\hline
\end{tabular}


Table I. Continued

\begin{tabular}{|c|c|c|c|c|c|c|}
\hline $\begin{array}{l}\text { I LOD } \\
\text { confidence } \\
\text { interval }\end{array}$ & $\begin{array}{l}\text { I LOD } \\
\text { confidence } \\
\text { interval } \\
\text { (cM) }\end{array}$ & $\begin{array}{l}\text { Max } \\
\text { LOD } \\
\text { (cM) }\end{array}$ & $\begin{array}{l}\text { Phenotype } \\
(\mu \mathrm{M})\end{array}$ & $\begin{array}{l}\text { No. of } \\
\text { genes } \\
\text { in I } \\
\text { LOD } \\
\text { interval }\end{array}$ & $\begin{array}{l}\text { Associated } \\
\text { gene; SNP } \\
(p \text { value })^{a}\end{array}$ & Function \\
\hline \multirow[t]{2}{*}{$9 q 31.3-q 33.3$} & $118-136$ & $1.59(126)$ & 80 & 122 & & \\
\hline & $118-134$ & $1.58(126)$ & 40 & 84 & & \\
\hline \multirow[t]{3}{*}{||$p|3-p| 2$} & $42-53$ & $2.54(46)$ & $\mathrm{IC}_{50}$ & 23 & $\begin{array}{l}C D 44 ; r s 7116432 \\
\left(1.51 \times 10^{-6}\right)\end{array}$ & Cell adhesion \\
\hline & & & & & $\begin{array}{l}\text { LRRC4C; } \\
\text { rs4375425 } \\
\left(3.24 \times 10^{-7}\right)\end{array}$ & $\begin{array}{l}\text { G } \\
\text { protein-mediated } \\
\text { signalling, cell } \\
\text { adhesion }\end{array}$ \\
\hline & & & & & $\begin{array}{l}\text { DKFZP586H2 I 23; } \\
\text { rsI7727535 } \\
\left(3.63 \times 10^{-12}\right)\end{array}$ & Serine protease \\
\hline \multirow[t]{4}{*}{ I Iq14.3-q23.3 } & $91-113$ & $3.36(99)$ & $\mathrm{IC}_{50}$ & 151 & $\begin{array}{l}\text { CNTN5; } \\
\text { rsI441382 } \\
\left(2.57 \times 10^{-7}\right)\end{array}$ & Cell adhesion \\
\hline & & & & & $\begin{array}{l}\text { IGSF4; rsI I II5437 } \\
\left(2.82 \times 10^{-7}\right)\end{array}$ & Cell adhesion \\
\hline & & & & & $\begin{array}{l}\text { PAKI; rs478I34 } \\
\left(1.07 \times 10^{-5}\right)\end{array}$ & $\begin{array}{l}\text { Protein serine/ } \\
\text { threonine kinase } \\
\text { activity }\end{array}$ \\
\hline & $94-117$ & 1.57 (109) & 10 & 208 & & \\
\hline |4q21.2-q24.। & $5 I-80$ & $1.64(66)$ & 10 & 134 & & \\
\hline
\end{tabular}

(Figure 4b). An evaluation of expression array data and qRT-PCR revealed significant correlation between the two methods $\left(r^{2}=0.60 ; \quad p=2 \times 10^{-4}\right)$. Although the GG genotype of the associated CD44 SNP, rs7116432, was associated with sensitivity to carboplatin, this SNP did not significantly explain the variation in baseline CD44 expression using CD44 expression data from exon array or qRT-PCR.

\section{siRNA-mediated knockdown of CD44}

Two cell lines with high CD44 mRNA expression and carboplatin $\mathrm{IC}_{50}$ values (GM06985 and GM11881) were chosen for CD44 siRNAmediated knockdown. Transfection efficiency 24 hours post-transfection was at least 70 per cent, when assessed by flow cytometry and qRT-PCR of siRNA directed against lamin A/C. qRT-PCR of CD44 in the two LCLs tested 24 hours posttransfection resulted in at least 90 per cent knockdown, as compared with non-targeting control. The most significant decrease in protein expression was at 48 hours, with less knockdown at 72 and 96 hours, for both cell lines (Figure 5a).

\section{Effect of CD44-siRNA on sensitivity to carboplatin}

Transfection with the CD44 siRNA resulted in a 23 per cent and 40 per cent reduction in $\mathrm{IC}_{50}$ compared with non-targeting siRNA groups for GM06985 $(p<0.01)$ and GM11881 $(p<0.05)$, 

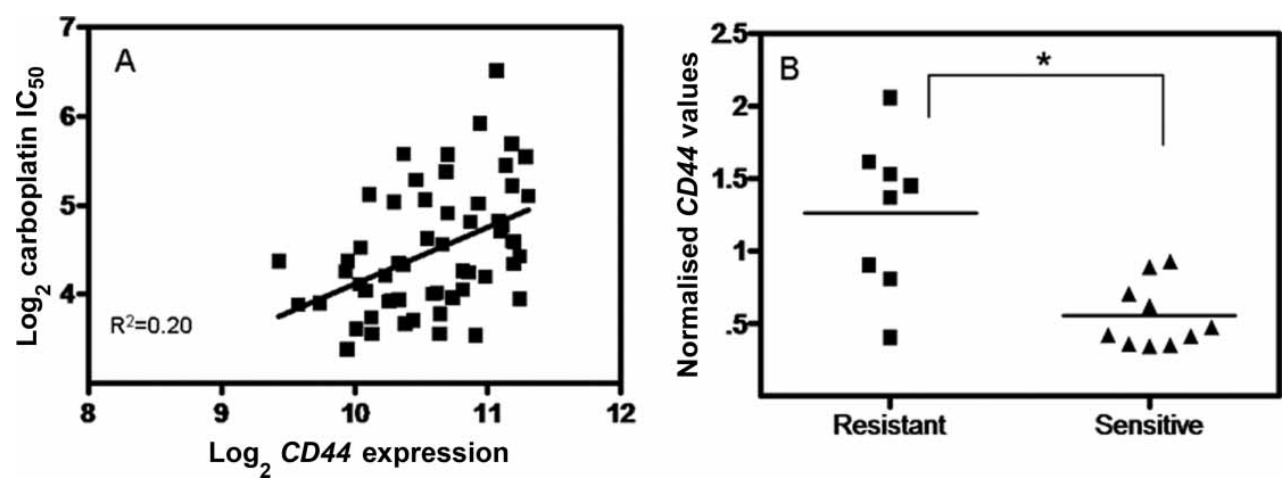

Figure 4. Baseline expression correlation of CD44 with carboplatin $I C_{50}$. (a) Linear regression of $\log _{2}$ transformed carboplatin $I C_{50}$ values on $\log _{2}$ transformed baseline CD44 expression (from exon expression array) and in 57 unrelated HapMap cell lines.

(b) Quantitative real-time polymerase chain reaction analysis of normalised CD44 expression in lymphoblastoid cell lines with the highest (resistant, $n=8$ ) and lowest (sensitive, $n=10$ ) carboplatin $I_{50}$ values. Each box represents biological triplicates run in duplicate $(* p<0.0$ I, Student's $t$-test).

respectively (Figure $5 b$ and $c$ ). To test the effects of experimental conditions and dose further, a mixed model analysis of variance was used to compare the two cytotoxicity curves for both cell lines. A significant interaction between dose and experimental condition was found $(p<0.01)$. These results indicate that the magnitude of the difference between the two conditions depended on the concentration of the drug. There was little difference between the two experimental conditions at less than $80 \mu \mathrm{M}$, with greater differences seen at concentrations of $\geq 80 \mu \mathrm{M}$. These differences reached statistical significance $(p<0.05)$ at the 80 and $160 \mu \mathrm{M}$ doses.

\section{Discussion}

The identification of pharmacogenetic variants contributing to variation in chemotherapeutic susceptibility is an area of great promise. In the present study, we utilised LCLs for genome-wide linkage and association analyses to identify genes and variants involved in carboplatin-induced cytotoxicity. Linkage-directed association identified SNPs within eight genes associated with carboplatin $\mathrm{IC}_{50}$, of which CD44 baseline expression explained 20 per cent of the variation in carboplatin $\mathrm{IC}_{50}$. Finally, the role of CD44 in resistance to carboplatin was validated using siRNA-mediated knockdown, resulting in greater sensitivity to carboplatin. Although cell-based approaches have been used to identify genetic variants contributing to chemotherapy, the validation of one such target using this approach is presented.

LCLs have been successfully used to identify genes involved in cytotoxicity, ${ }^{13-17,21}$ alternative splicing, ${ }^{22}$ expression $^{23-25}$ and other complex traits. ${ }^{26-28}$ Furthermore, LCLs have been used to study population and gender effects on quantitative traits. ${ }^{29-31}$ Consistent with cisplatin-, daunorubicin-, docetaxel- and 5-fluorouracil-induced cytotoxicity in LCLs, ${ }^{13,14,17,21}$ carboplatin cytototoxic phenotypes are significantly heritable. Realising that genetics contributes significantly to susceptibility to chemotherapeutic-induced cytotoxicity, our laboratory has developed two cell-based models for identifying genetic variants contributing to chemotherapeutic induced cytotoxicity. One model is a sequential approach that integrates SNP genotype, gene expression and chemotherapeutic cytotoxicity. ${ }^{15,16}$ Specifically, a whole-genome association is performed between HapMap SNP genotypes and cytotoxicity, followed by the use of those SNPs identified as being associated with cytotoxicity in a second association with gene expression. Lastly, linear regression is performed between gene expression of the 'target gene' and cytotoxicity to identify genes whose expression correlates with chemotherapeutic cytotoxicity. The second cellbased model is presented in this paper, in which large pedigrees are used to determine heritability, followed by the identification of regions in 


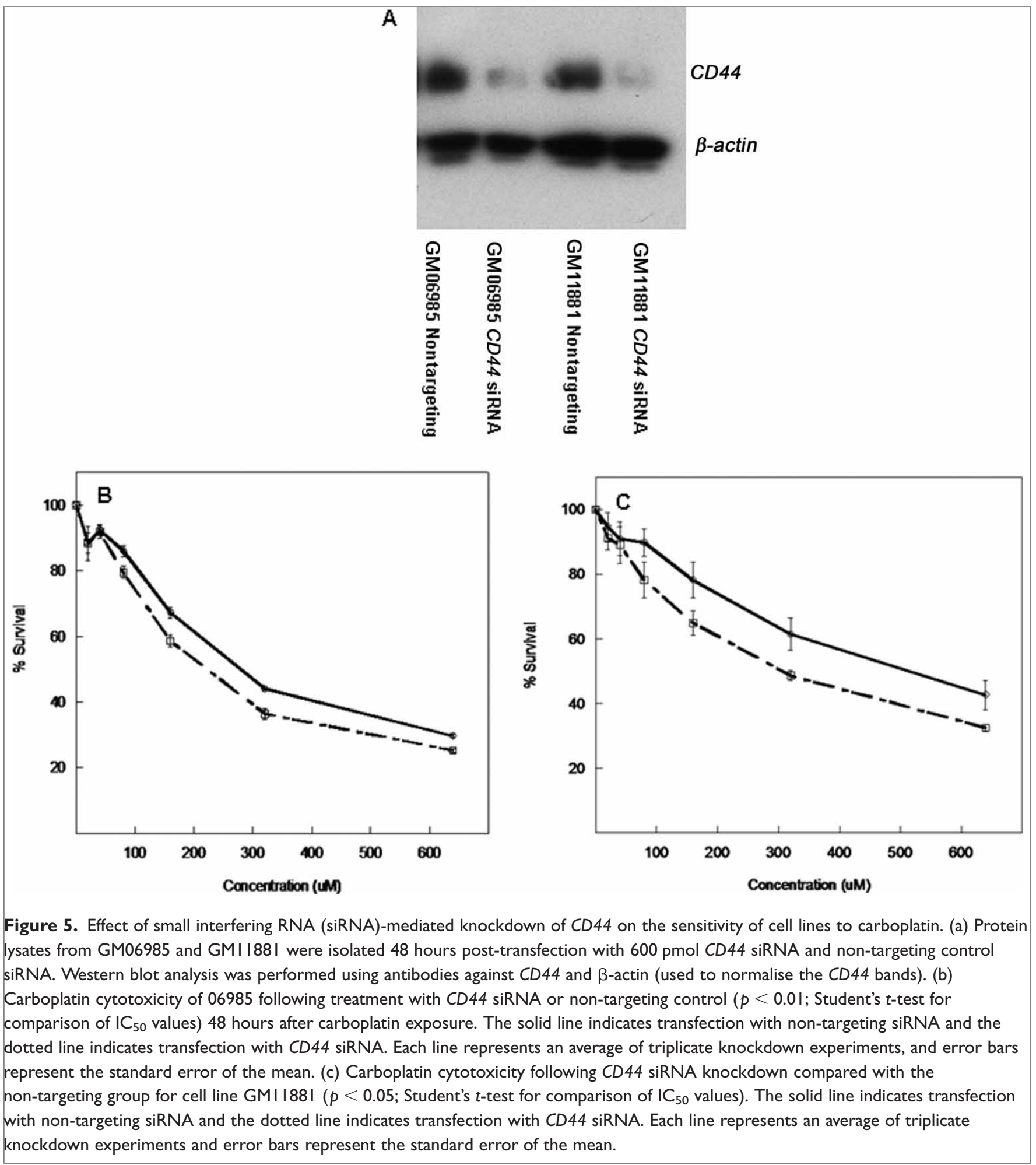

the genome that harbour genetic variation that explains human variation in drug-induced cytotoxicity. To narrow down the list of genetic variants within suggestive LOD peaks, an association analysis on SNPs within 1 LOD confidence interval is evaluated in a subset of samples containing dense genotypic data. This approach was applied to daunorubicin ${ }^{14}$ and cisplatin. ${ }^{17}$ These approaches 
can be used as a springboard for further analysis and validation of candidate SNPs in other model systems and, ultimately, clinical trials. Some improvements in this model include the utilisation of more CEPH pedigrees (ie 34 compared with previous studies using 24 to 27 pedigrees ${ }^{13,14}$ ) and the use of ANOVA analysis on unrelated HapMap and Perlegen LCLs instead of the quantitative transmission disequilibrium test on 30 trios, providing flexibility to test different genetic models for each SNP. The current study incorporated an analysis of gene expression to identify the contribution of baseline gene expression of the associated genes to variation in drug sensitivity. Lastly, this study included functional analysis by siRNA-mediated knockdown studies to validate results from the whole-genome approach. One interesting observation was the lack of overlap in genetic variants associated with carboplatinand cisplatin-induced ${ }^{17}$ cytotoxicity using a linkagedirected association analysis. Twenty-seven and 34 families were analysed for cisplatin and carboplatin susceptibility, respectively. These drugs have different toxicity profiles, and this model was designed to be more relevant to drug toxicity than tumour response. Nonetheless, one might expect some overlap between the findings for these similarly acting drugs. Therefore, resequencing in candidate genes will be performed in future studies. In addition, we will perform a directed study to evaluate the degree to which genes found for one drug contribute to the other drug, as we did with CD44 in this analysis.

We tested 68 SNPs from the HapMap and Perlegen samples in the CD44 gene locus, in which 54 of these SNPs had minor allele frequencies greater than 5 per cent for association with carboplatin $\mathrm{IC}_{50}$ or CD44 expression. Our linkagedirected association identified rs7116432 an intronic SNP located in CD44 - as being associated with the carboplatin $\mathrm{IC}_{50}(p=1.5 \times$ $\left.10^{-6} ; \mathrm{FDR}=0.003\right)$. This SNP was not associated with the variation in expression of CD44 or any other genes, as measured on the Affymetrix exon array; however, this particular SNP may contribute to an induction in CD44 gene expression after carboplatin treatment. In addition, there were two SNPs, rs7116739 and rs11607862, which were in strong linkage disequilibrium with rs7116432. These SNPs were not significantly associated with carboplatin $\mathrm{IC}_{50}$, however, and did not associate with CD44 baseline expression. It is notable that a SNP, rs7894637, located in TCF8 on chromosome 10 , significantly explained 25 per cent of the variation in baseline CD44 expression $\left(p=2 \times 10^{-6}\right)$. This SNP is not within 1 confidence interval of any LOD peaks $>1.5$ from the linkage scan, however, and is not associated with the expression of other genes (data not shown). Future studies will focus more on the causal variants and haplotypes affecting CD44 function and their role in carboplatin resistance.

Proteins from one CD44 family are involved in a wide variety of cellular processes, such as cell adhesion, cell survival, regulation of growth, invasion and proliferation. ${ }^{32,33}$ CD44 is a transmembrane glycoprotein involved in the adaptation of cells to their microenvironment. ${ }^{33}$ CD 44(s), which is the smallest standard isoform, is expressed in the majority of vertebrate tissues, ${ }^{32}$ whereas the alternatively spliced transcripts are expressed in proliferating cells and many cancers. ${ }^{33}$ Regarding treatment outcome, a recent haplotype derived from three intronic SNPs and one SNP in the $3^{\prime}$ untranslated region in CD44 was associated with an increased risk of early adverse skin reactions after radiotherapy in breast cancer patients. ${ }^{34}$ CD 44 has also been implicated in resistance (including resistance to apoptosis) to various chemotherapeutic agents, including alkylating agents, platinating agents, etoposide and dexamethasone. ${ }^{35-41}$ One study revealed that enhanced expression of a CD44 variant isoform was associated with 1,3-bis(2chloroethyl)-1-nitrosourea (BCNU) resistance in colon cancer lines. ${ }^{42}$ More specifically, hyaluronan (HA)-CD44 signalling has also been implicated in cellular resistance to cisplatin and methotrexate in head and neck cancer cells and altered adriamycin sensitivity in breast cancer. $^{40,41,43,44}$ Previous studies performed in tumour cell lines suggest that epidermal growth factor receptor (EGFR)mediated downstream signalling events resulting from the HA-CD44 interaction is a likely cause of resistance. ${ }^{39-41}$ In this model, HA binding to 
CD44 promotes its association with, and phosphorylation of, EGFR, which signals the downstream phosphorylation of mitogen-activated protein kinases to promote chemotherapy resistance. In addition, HA-CD44 promotes other intracellular signalling cascades through its interaction with phospholipase C-gamma-1, phosphoinositide 3-kinase, Nanog-Stat3 and other pathways. $^{39-41,45-48}$

Overexpression of CD44 has been shown to confer an anti-apoptotic effect following treatment with etoposide in colon cancer cells ${ }^{36}$ and after treatment with dexamethasone in $\mathrm{T}$ lymphocytes. ${ }^{49}$ Murine colon epithelium cells deficient in CD44 were assessed for apoptosis after total body irradiation. Upregulation of apoptosis-associated genes, such as the genes encoding caspases 3 and 9, indicated that a mitochondrial pathway was involved in apoptosis. ${ }^{37}$ In the current linkage study, we observed several caspases (1, 4, 5 and 12) within a high LOD score region on chromosome 11 $(\mathrm{LOD}=3.36)$ for the $\mathrm{IC}_{50}$ phenotype. Genetic variation within the caspases, along with CD44, could act in concert to increase resistance to carboplatin. In addition, a conserved intronic SNP across multiple species, rs17727535 (located in DKFZP586H2123), was the SNP most significantly associated with carboplatin $\mathrm{IC}_{50}\left(p=3.63 \times 10^{-12}\right.$; FDR <0.001). This gene is located underneath the same linkage peak as CD44 and has serine protease activity. Maquarre et al. ${ }^{50}$ recently demonstrated that ligation of CD44 by a monoclonal antibody in leukaemia cells induced apoptosis through a caspaseand serine protease-dependent pathway. Thus, these two pathways may act in concert to confer resistance to carboplatin. Taken together, overexpression of CD44 may mediate signalling, which can lead to resistance to carboplatin, and possibly to other co-administered drugs, by conferring an anti-apoptotic effect. One could consider CD44 expression levels as a biomarker of resistance to carboplatin.

It is important to note that cytotoxicity is a multigenic trait, and there are many genes whose expression levels may be contributing to carboplatin $\mathrm{IC}_{50}$ values and drug resistance. Since PAK1 expression also showed a significant contribution to carboplatin $\mathrm{IC}_{50}$, the effect of knocking down PAK1 with CD44 and/or other genes might be considered.

Our unbiased genome-wide linkage-directed association analyses, employing LCLs, identified previously unknown genes and variants involved in carboplatin-induced cytotoxicity. The use of LCLs enabled us to demonstrate the involvement of CD44 expression in resistance to carboplatin. Overall, the experimental data elucidating the contribution of CD44 to carboplatin resistance in LCLs encourages the use of this sequential wholegenome approach. In addition, all eight genes could be further examined without a focus on gene expression levels, since their contribution to susceptibility to cytotoxicity could be through other means, such as post-translational modifications or alternative splicing. Despite the identification of interesting genes and variants through our genetic analyses, there are many well-studied genes contributing to chemotherapeutic resistance and toxicity that were not identified through linkage analysis.

There were two caveats in our association study. First, we were limited to the SNPs within the International HapMap; therefore SNPs in candidate genes may not be well represented. Secondly, 30 trios (90 samples) may lack the power to detect some candidate genetic variants. On average, the heritability estimates ranged from 17-35 per cent in our pedigree study. Candidate genes that have low or modest contributions to cytotoxicity or lack genetic variation may not be found in the final gene list. Future experiments will include siRNA-mediated knockdown of candidate genes whose expression may contribute to resistance to carboplatin. Finally, genes and variants identified through our approach should be also tested with regard to chemotherapeutic susceptibility in appropriate tumour cell lines. One could consider this whole-genome approach to identify genes and mechanisms involved in other cellular phenotypes.

\section{Acknowledgments}

This Pharmacogenetics of Anticancer Agents Research (PAAR) Group (http://pharmacogenetics.org) study was supported by NIH/NIGMS grant U01GM61393 and data deposits are supported by U01GM61374 (http:// 
pharmgkb.org/). This study was also supported by P50CA125183 University of Chicago Breast Cancer SPORE grant. We are grateful for excellent technical support provided by Dr Jeong-Ah Kang in maintaining the cell lines. We are also thankful to Dr Blanca Camoretti-Mercado for intellectual contributions and use of the Amaxa Nucleofector device.

Data deposits: Phenotype data (PS207015) is deposited into (http://www.pharmgkb.org/). The gene expression data described in this paper have been deposited into GEO (GenBank Accession No: GSE7851).

\section{References}

1. Ho, Y.P., Au-Yeung, S.C. and To, K.K. (2003), 'Platinum-based anticancer agents: Innovative design strategies and biological perspectives', Med. Res. Rev. Vol. 23, pp. 633-655.

2. Kelland, L. (2007), 'The resurgence of platinum-based cancer chemotherapy', Nat. Rev. Cancer Vol. 7, pp. 573-584.

3. Efferth, T. and Volm, M. (2005), 'Pharmacogenetics for individualized cancer chemotherapy', Pharmacol. Ther. Vol. 107, pp. 155-176.

4. Wang, D. and Lippard, S.J. (2005), 'Cellular processing of platinum anticancer drugs', Nat. Rev. Drug Discov. Vol. 4, pp. 307-320.

5. Brabec, V. and Kasparkova, J. (2005), 'Modifications of DNA by platinum complexes. Relation to resistance of tumors to platinum antitumor drugs', Drug Resist. Updat. Vol. 8, pp. 131-146.

6. Stewart, D.J. (2007), 'Mechanisms of resistance to cisplatin and carboplatin', Crit. Rev. Oncol. Hematol. Vol. 63, pp. 12-31.

7. Vekris, A., Meynard, D., Haaz, M.C. et al. (2004), 'Molecular determinants of the cytotoxicity of platinum compounds: The contribution of in silico research', Cancer Res. Vol. 64, pp. 356-362.

8. Rabik, C.A. and Dolan, M.E. (2007), 'Molecular mechanisms of resistance and toxicity associated with platinating agents', Cancer Treat. Rev. Vol. 33, pp. 9-23.

9. Suk, R., Gurubhagavatula, S., Park, S. et al. (2005), 'Polymorphisms in ERCC1 and grade 3 or 4 toxicity in non-small cell lung cancer patients', Clin. Cancer Res. Vol. 11, pp. 1534-1538.

10. Olaussen, K.A., Dunant, A., Fouret, P. et al. (2006), 'DNA repair by ERCC1 in non-small-cell lung cancer and cisplatin-based adjuvant chemotherapy', N. Engl. J. Med. Vol. 355, pp. 983-991.

11. Olaussen, K.A., Mountzios, G. and Soria, J.C. (2007), 'ERCC1 as a risk stratifier in platinum-based chemotherapy for nonsmall-cell lung cancer', Curr. Opin. Pulm. Med. Vol. 13, pp. 284-289.

12. Bewick, M.A., Conlon, M.S. and Lafrenie, R.M. (2006), 'Polymorphisms in XRCC1, XRCC3, and CCND1 and survival after treatment for metastatic breast cancer', J. Clin. Oncol. Vol. 24, pp. $5645-5651$.

13. Dolan, M.E., Newbold, K.G., Nagasubramanian, R. et al. (2004), 'Heritability and linkage analysis of sensitivity to cisplatin-induced cytotoxicity', Cancer Res. Vol. 64, pp. 4353-4356.

14. Duan, S., Bleibel, W.K., Huang, R.S. et al. (2007), 'Mapping genes that contribute to daunorubicin-induced cytotoxicity', Cancer Res. Vol. 67, pp. 5425-5433.

15. Huang, R.S., Duan, S., Bleibel, W.K. et al. (2007), 'A genome-wide approach to identify genetic variants that contribute to etoposide-induced cytotoxicity', Proc. Natl. Acad. Sci. USA Vol. 104, pp. 9758-9763.

16. Huang, R.S., Duan, S., Shukla, S.J. et al. (2007), 'Identification of genetic variants contributing to cisplatin-induced cytotoxicity by use of a genomewide approach', Am. J. Hum. Genet. Vol. 81, pp. 427-437.

17. Shukla, S.J., Duan, S., Badner, J.A., Wu, X. and Dolan, M.E. (2008), 'Susceptibility loci involved in cisplatin-induced cytotoxicity and apoptosis', Pharmacogenet. Genomics Vol. 18, pp. 253-262.

18. Almasy, L. and Blangero, J. (1998), 'Multipoint quantitative-trait linkage analysis in general pedigrees', Am. J. Hum. Genet. Vol. 62, pp. 1198-1211.
19. Abecasis, G.R., Cherny, S.S., Cookson, W.O. and Cardon, L.R. (2002), 'Merlin — Rapid analysis of dense genetic maps using sparse gene flow trees', Nat. Genet. Vol. 30, pp. 97-101.

20. Zhang, W., Duan, S., Kistner, E.O. et al. (2008), 'Evaluation of genetic variants contributing to differences in gene expression', Am. J. Hum. Genet. Vol. 82, pp. 631-640.

21. Watters, J.W., Kraja, A., Meucci, M.A., Province, M.A. and McLeod, H.L. (2004), 'Genome-wide discovery of loci influencing chemotherapy cytotoxicity', Proc. Natl. Acad. Sci. USA Vol. 101, pp. 11809-11814.

22. Kwan, T., Benovoy, D., Dias, C. et al. (2007), 'Heritability of alternative splicing in the human genome', Genome Res. Vol. 17, pp. 1210-1218.

23. Dixon, A.L., Liang, L., Moffatt, M.F. et al. (2007), 'A genome-wide association study of global gene expression', Nat. Genet. Vol. 39, pp. 1202-1207.

24. Morley, M., Molony, C.M., Weber, T.M. et al. (2004), 'Genetic analysis of genome-wide variation in human gene expression', Nature Vol. 430, pp. $743-747$.

25. Stranger, B.E., Forrest, M.S., Dunning, M. et al. (2007), 'Relative impact of nucleotide and copy number variation on gene expression phenotypes', Science Vol. 315, pp. 848-853.

26. Baron, C.A., Liu, S.Y., Hicks, C. and Gregg, J.P. (2006), 'Utilization of lymphoblastoid cell lines as a system for the molecular modeling of autism', J. Autism Dev. Disord. Vol. 36, pp. 973-982.

27. Hu, V.W., Frank, B.C., Heine, S., Lee, N.H. and Quackenbush, J. (2006), 'Gene expression profiling of lymphoblastoid cell lines from monozygotic twins discordant in severity of autism reveals differential regulation of neurologically relevant genes', BMC Genomics Vol. 7, p. 118.

28. Nishimura, Y., Martin, C.L., Vazquez-Lopez, A. et al. (2007), 'Genome-wide expression profiling of lymphoblastoid cell lines distinguishes different forms of autism and reveals shared pathways', Hum. Mol. Genet. Vol. 16, pp. 1682-1698.

29. Huang, R.S., Kistner, E.O., Bleibel, W.K., Shukla, S.J. and Dolan, M.E. (2007), 'Effect of population and gender on chemotherapeutic agent-induced cytotoxicity', Mol. Cancer Ther. Vol. 6, pp. 31-36.

30. Weiss, L.A., Pan, L., Abney, M. and Ober, C. (2006), 'The sex-specific genetic architecture of quantitative traits in humans', Nat. Genet. Vol. 38, pp. 218-222.

31. Zhang, W., Bleibel, W.K., Roe, C.A., Cox, N.J. and Dolan, M.E. (2007), 'Gender-specific differences in expression in human lymphoblastoid cell lines', Pharmacogenet. Genomics Vol. 17, pp. 447-450.

32. Cheng, C., Yaffe, M.B. and Sharp, P.A. (2006), 'A positive feedback loop couples Ras activation and CD44 alternative splicing', Genes Dev. Vol. 20, pp. 1715-1720.

33. Ponta, H., Sherman, L. and Herrlich, P.A. (2003), 'CD44: From adhesion molecules to signalling regulators', Nat. Rev. Mol. Cell Biol. Vol. 4, pp. 33-45.

34. Suga, T., Ishikawa, A., Kohda, M. et al. (2007), 'Haplotype-based analysis of genes associated with risk of adverse skin reactions after radiotherapy in breast cancer patients', Int. J. Radiat. Oncol. Biol. Phys. Vol. 69, pp. 685-693.

35. Hagel, C., Park, S.H., Puchner, M.J. and Stavrou, D. (2004), 'CD44 expression and tumour cell density correlate with response to tamoxifen/ carboplatin chemotherapy in glioblastomas', J. Neurooncol. Vol. 66 pp. 139-146.

36. Lakshman, M., Subramaniam, V., Rubenthiran, U. and Jothy, S. (2004), 'CD44 promotes resistance to apoptosis in human colon cancer cells', Exp. Mol. Pathol. Vol. 77, pp. 18-25.

37. Lakshman, M., Subramaniam, V., Wong, S. and Jothy, S. (2005), 'CD44 promotes resistance to apoptosis in murine colonic epithelium', J. Cell Physiol. Vol. 203, pp. 583-588.

38. Ohwada, C., Nakaseko, C., Koizumi, M. et al. (2008), 'CD44 and hyaluronan engagement promotes dexamethasone resistance in human myeloma cells', Eur. J. Haematol. Vol. 80, pp. 245-250.

39. Wang, S.J. and Bourguignon, L.Y. (2006), 'Hyaluronan and the interaction between CD44 and epidermal growth factor receptor in oncogenic signaling and chemotherapy resistance in head and neck cancer', Arch. Otolaryngol. Head Neck Surg. Vol. 132, pp. 771-778. 
40. Wang, S.J. and Bourguignon, L.Y. (2006), 'Hyaluronan-CD44 promotes phospholipase $\mathrm{C}$-mediated $\mathrm{Ca} 2+$ signaling and cisplatin resistance in head and neck cancer', Arch. Otolaryngol. Head Neck Surg. Vol. 132, pp. 19-24.

41. Wang, S.J., Peyrollier, K. and Bourguignon, L.Y. (2007), 'The influence of hyaluronan-CD44 interaction on topoisomerase II activity and etoposide cytotoxicity in head and neck cancer', Arch. Otolaryngol. Head Neck Surg. Vol. 133, pp. 281-288.

42. Bates, R.C., Edwards, N.S., Burns, G.F. and Fisher, D.E. (2001), 'A CD44 survival pathway triggers chemoresistance via lyn kinase and phosphoinositide 3-kinase/Akt in colon carcinoma cells', Cancer Res. Vol. 61, pp. 5275-5283.

43. Misra, S., Ghatak, S. and Toole, B.P. (2005), 'Regulation of MDR1 expression and drug resistance by a positive feedback loop involving hyaluronan, phosphoinositide 3-kinase, and ErbB2', J. Biol. Chem. Vol. 280, pp. 20310-20315.

44. Misra, S., Ghatak, S., Zoltan-Jones, A. and Toole, B.P. (2003), 'Regulation of multidrug resistance in cancer cells by hyaluronan', J. Biol. Chem. Vol. 278, pp. 25285-25288.
45. Entwistle, J., Hall, C.L. and Turley, E.A. (1996), 'HA receptors: Regulators of signalling to the cytoskeleton', J. Cell Biochem. Vol. 61, pp. $569-577$

46. Lin, Y.H. and Yang-Yen, H.F. (2001), 'The osteopontin-CD44 survival signal involves activation of the phosphatidylinositol 3-kinase/Akt signaling pathway', J. Biol. Chem. Vol. 276, pp. 46024-46030.

47. Turley, E.A., Noble, P.W. and Bourguignon, L.Y. (2002), 'Signaling properties of hyaluronan receptors', J. Biol. Chem. Vol. 277, pp. 4589-4592.

48. Bourguignon, L.Y., Peyrollier, K., Xia, W. and Gilad, E. (2008), 'Hyaluronan-CD44 interaction activates stem cell marker, nanog, stat-3mediated MDR1 gene expression and ankyrin-regulated multidrug efflux in breast and ovarian tumor cells', J. Biol. Chem. vol. 283, pp. $17635-17651$.

49. Ayroldi, E., Cannarile, L., Migliorati, G. et al. (1995), 'CD44 (Pgp-1) inhibits CD3 and dexamethasone-induced apoptosis', Blood. Vol. 86, pp. 2672-2678.

50. Maquarre, E., Artus, C., Gadhoum, Z. et al. (2005), 'CD44 ligation induces apoptosis via caspase- and serine protease-dependent pathways in acute promyelocytic leukemia cells', Leukemia Vol. 19, pp. 2296-2303.

\section{Supplementary Table I}

\begin{tabular}{|c|c|c|c|c|c|}
\hline $\begin{array}{l}\text { Single nucleotide } \\
\text { polymorphism }\end{array}$ & Chromosome & Position (bp) & Gene & $p$ value & $\begin{array}{c}\text { False discovery } \\
\text { rate }(\%)\end{array}$ \\
\hline rsII784I5I & 8 & 104275289 & $B A A L C$ & $3.6 \times 10^{-5}$ & 2.7 \\
\hline rs I 3255229 & 8 & 104279675 & $B A A L C$ & $1.0 \times 10^{-5}$ & 0.9 \\
\hline rs2201369 & 8 & 104291201 & BAALC & $9.1 \times 10^{-11}$ & 0.0 \\
\hline rsI7799574 & 8 & 104298709 & BAALC & $9.1 \times 10^{-11}$ & 0.0 \\
\hline rs|7378|30 & 8 & 124310523 & C8orf76 & $1.5 \times 10^{-5}$ & 1.2 \\
\hline rs7।I6432 & II & 35200634 & CD44 & $1.5 \times 10^{-6}$ & 0.3 \\
\hline rs I0790959 & II & $99|5204|$ & CNTN5 & $1.5 \times 10^{-6}$ & 0.3 \\
\hline rs|44|382 & II & 99198199 & CNTN5 & $2.6 \times 10^{-7}$ & 0.1 \\
\hline rs I7727535 & II & 35501279 & DKFZP586H2 I 23 & $3.6 \times 10^{-12}$ & 0.0 \\
\hline rs II2I5437 & II & II 4609382 & IGSF4 & $2.8 \times 10^{-7}$ & 0.1 \\
\hline rs4375425 & II & 40183956 & LRRC4C & $3.2 \times 10^{-7}$ & 0.1 \\
\hline rs476925 & II & 76793285 & PAKI & $1.1 \times 10^{-5}$ & 0.9 \\
\hline rs538670 & II & 76766226 & PAKI & $1.1 \times 10^{-5}$ & 0.9 \\
\hline rs478I34 & II & 76753207 & PAKI & $1.1 \times 10^{-5}$ & 0.9 \\
\hline rs495889 & II & 76796364 & PAKI & $1.1 \times 10^{-5}$ & 0.9 \\
\hline rs628325 & II & $768 \mid 4368$ & PAKI & $1.1 \times 10^{-5}$ & 0.9 \\
\hline rs3019258 & II & 76836767 & PAKI & $1.1 \times 10^{-5}$ & 0.9 \\
\hline rs674652 & II & 76785476 & PAKI & $1.1 \times 10^{-5}$ & 0.9 \\
\hline
\end{tabular}

